\title{
An Integral Involving Certain General Class of Polynomials and the Special Functions
}

Ashok Singh Shekhawat ${ }^{*}$ Parul Gupta ${ }^{* *}$ and Rakeshwar Purohit***

*Department of Mathematics, Arya College of Engineering and Information Technology Jaipur, Rajasthan

*** Department of Mathematics, Arya Institute of Engineering and Technology Jaipur, Rajasthan

***Department of Mathematics and Statistics, University College of Science, Mohanlal Sukhadia University, Udaipur, Rajasthan

Abstract: The object of present paper is to establish an integral pertaining to a product of Fox's H-function, general polynomials given by Srivastava and $H$-function of several complex variables given by Srivastava and Panda with general arguments of quadratic nature.

\section{Introduction}

(a). The H-function of several complex variables is defined by Srivastava and Panda as:

$$
\left.\begin{array}{r}
H\left[p_{1}, \ldots, p_{r}\right]=H_{A+1, C+1:\left[B^{\prime}, D^{\prime}\right] ; \ldots ;\left[B^{(r)}, D^{(r)}\right]}^{0, \lambda+1:\left(u^{(1)}, v^{(1)}\right) ; .\left(u^{(r)}, v^{(r)}\right)}\left[\begin{array}{l}
{\left[\left(\beta_{1}\right): \theta^{\prime} ; \ldots ; \theta^{(r)}\right]:\left[\left(\beta_{2}^{(1)}\right): \phi^{\prime}\right] ; \ldots ;\left[\left(\beta_{2}^{(r)}: \phi^{(r)}\right] ;\right.} \\
\left.\left(\beta_{3}\right): \psi^{\prime} ; \ldots ; \psi^{(r)}\right]:\left[\left(\beta_{1}\right)^{(1)}: \delta^{\prime}\right] ; \ldots ;\left[\left(\beta_{4}^{(r)}\right): \rho^{(r)}\right] ;
\end{array} p_{1}, \ldots, p_{r}\right.
\end{array}\right]
$$

(b). The series representation of Fox's H-function is given by

$$
H_{P, Q}^{M, N}\left[p \mid \begin{array}{l}
\left(e_{P}, F_{P}\right) \\
\left({ }_{Q}, F_{Q}\right)
\end{array}\right]=\sum_{G=0}^{\infty} \sum_{g=1}^{M} \frac{(-1)^{G}}{G ! F_{g}} \xi\left(\rho_{G}\right) Z^{\rho_{G}}
$$

where

$$
\begin{aligned}
& \prod_{j=1}^{M} \Gamma\left(f_{i}-F_{j} \rho_{G}\right) \prod_{j=1}^{N} \Gamma\left(1-e_{j}+E_{j} \rho_{G}\right) \\
& \xi\left(\rho_{\mathrm{G}}\right)=\frac{j \neq \mathrm{g}}{\mathrm{Q}} \\
& \left.\prod_{j=M+1} \Gamma 1-f_{j}+F_{j} \rho_{G}\right) \prod_{j=N+1} \Gamma\left(e_{j}-E_{j} \rho_{G}\right)
\end{aligned}
$$

and

$$
\delta_{G}=\left(\frac{f_{j}+G}{F_{g}}\right)
$$

(c). Srivastava has defined and introduced the general polynomials as

$$
\begin{gathered}
S_{N_{1}, \ldots, N_{s}}^{M_{1}, \ldots, M_{s}}\left[w_{1}, \ldots, w_{s}\right]=\sum_{\mu_{1}=0}^{\left[N_{1} / M_{1}\right]} \cdots \sum_{\mu_{s}=0}^{\left[N_{s} / M_{s}\right]} \frac{\left(-N_{1}\right)_{M_{1} \mu_{1}}}{\mu_{1} !} \frac{\left(-N_{s}\right)_{M_{s} \mu_{s}}}{\mu_{s} !} \\
. A\left[N_{1}, \mu_{1} ; \ldots ; N_{s}, \mu_{s}\right] w_{1}^{\mu_{1}} \ldots w_{s}^{\mu_{s}}
\end{gathered}
$$

where $\mathrm{N}_{\mathrm{i}}=0,1,2, \ldots, \forall \mathrm{i}(1, \ldots, \mathrm{s}), \mathrm{M}_{1}, \ldots, \mathrm{M}_{\mathrm{s}}$ are arbitrary positive integers and the coefficients $\mathrm{A}\left[\mathrm{N}_{1}, \mu_{1} ; \ldots ; \mathrm{N}_{\mathrm{s}}, \mu_{\mathrm{s}}\right]$ are arbitrary constants, real or complex.

(d). A general class of multivariable polynomial defined and represented by Srivastava and Garg, is given in the following manner 
$\mathrm{S}_{\mathrm{V}}^{\mathrm{W}_{1}, \ldots, \mathrm{w}_{\mathrm{s}}}\left[\mathrm{a}_{1}, \ldots, \mathrm{a}_{\mathrm{s}}\right]=\sum_{\mathrm{k}_{1}, \ldots, \mathrm{k}_{\ell}=0}^{\mathrm{w}_{1} \mathrm{k}_{1}+\ldots+\mathrm{w}_{\ell} \mathrm{k}_{\ell}}(-\mathrm{V})_{\mathrm{w}_{1} \mathrm{k}_{1}+\ldots+\mathrm{w}_{\ell} \mathrm{k}_{\ell}} \mathrm{A}\left(\mathrm{V}: \mathrm{k}_{1}, \ldots, \mathrm{k}_{\ell}\right) \frac{\mathrm{y}_{1}^{\mathrm{k}_{1}}}{\mathrm{k}_{1} !}, \ldots, \frac{\left(\mathrm{y}_{\mathrm{s}}\right)^{\mathrm{k}_{\ell}}}{\mathrm{k}_{\ell} !}$

II. The Main Result

The following integral has been established in this paper

$$
\begin{aligned}
& \int_{0}^{\infty} \mathrm{x}^{1-\mu}\left(\beta_{1}+\beta_{2} \mathrm{x}+\beta_{3} \mathrm{x}^{2}\right)^{\mu-3 / 2} \mathrm{H}_{\mathrm{P}, \mathrm{Q}}^{\mathrm{M}, \mathrm{N}}\left[\left(\frac{\mathrm{x}}{\beta_{1}+\beta_{2} \mathrm{x}+\beta_{3} \mathrm{x}^{2}}\right)^{v} \mid \begin{array}{c}
\left(\mathrm{e}_{\mathrm{P}}, \mathrm{E}_{\mathrm{P}}\right) \\
\left(\mathrm{f}_{\mathrm{Q}}, \mathrm{F}_{\mathrm{Q}}\right)
\end{array}\right] \\
& . S_{N_{1}, \ldots, N_{s}}^{M_{1}, \ldots, M_{s}}\left[v_{1}\left(\frac{x}{\beta_{1}+\beta_{2} x+\beta_{3} x^{2}}\right)^{d_{1}}, \ldots, v_{s}\left(\frac{x}{\beta_{1}+\beta_{2} x+\beta_{3} x^{2}}\right)^{d_{s}}\right] \\
& . \mathrm{S}_{\mathrm{V}}^{\mathrm{w}_{1}, \ldots, \mathrm{w}_{\ell}}\left[\mathrm{y}_{1}\left(\frac{\mathrm{x}}{\beta_{1}+\beta_{2}+\beta_{3} \mathrm{x}^{2}}\right)^{\mathrm{m}_{1}}, \ldots, \mathrm{y}_{\ell}\left(\frac{\mathrm{x}}{\beta_{1}+\beta_{2} \mathrm{x}+\beta_{3} \mathrm{x}^{2}}\right)^{\mathrm{m}_{\ell}}\right] \\
& H\left[p_{1}\left(\frac{x}{\beta_{1}+\beta_{2} x+\beta_{3} x^{2}}\right)^{v_{1}}, \ldots, p_{r}\left(\frac{x}{\beta_{1}+\beta_{2} x+\beta_{3} x^{2}}\right)^{v_{r}}\right] d x \\
& =\sqrt{\frac{\pi}{\beta_{3}}} \sum_{\mathrm{G}=0}^{\infty} \sum_{\mathrm{g}=1}^{\mathrm{m}} \sum_{\mu_{1}=0}^{\left[\mathrm{N}_{1} / \mathrm{M}_{1}\right]} \cdots \sum_{\mu_{\mathrm{s}}=0}^{\left[\mathrm{N}_{\mathrm{s}} / \mathrm{M}_{\mathrm{s}}\right]} \frac{(-1)^{\mathrm{G}}}{\mathrm{G} ! \mathrm{F}_{\mathrm{g}}} \frac{\left(-\mathrm{N}_{1}\right)_{\mathrm{M}_{1} \mu_{1}}}{\mu_{1} !} \ldots \frac{\left(-\mathrm{N}_{\mathrm{s}}\right)_{\mathrm{M}_{\mathrm{s}} \mu_{\mathrm{s}}}}{\mu_{\mathrm{s}} !} \xi\left(\rho_{\mathrm{G}}\right)
\end{aligned}
$$

$$
\mathrm{A}\left[\mathrm{N}_{1} \mu_{1} ; \ldots ; \mathrm{N}_{\mathrm{s}} \mu_{\mathrm{s}}\right] \mathrm{v}_{1}^{\mu_{1}}, \ldots, \mathrm{v}_{\mathrm{s}}^{\mu_{\mathrm{s}}} \mathrm{A}\left(\mathrm{V} ; \mathrm{k}_{1}, \ldots, \mathrm{k}_{\ell}\right)
$$$$
\left(\beta_{2}+\sqrt{\beta_{1} \beta_{3}}\right){ }^{\left.\mu-v \rho_{G}-\sum_{i=1}^{s} d_{i} \mu_{i}-\sum_{i=1}^{\ell} m_{i} k_{i}-1\right)}
$$

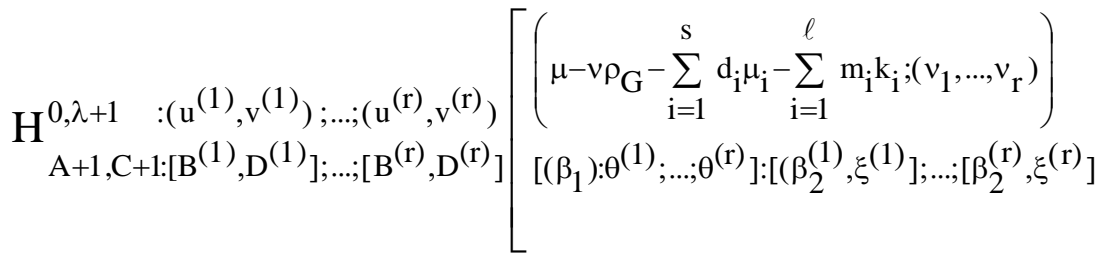

$\left[\left(\beta_{3}\right) ; \xi^{(1)}, \ldots, \xi^{(r)}\right]\left[\mu-v \rho_{\mathrm{G}}-\sum_{\mathrm{i}=1}^{\mathrm{s}} \mathrm{d}_{\mathrm{i}} \mu_{\mathrm{i}}-\sum_{\mathrm{i}=1}^{\ell} \mathrm{m}_{\mathrm{i}} \mathrm{k}_{\mathrm{i}}-1 / 2 ; v_{1}, \ldots, v_{\mathrm{r}}\right]:$
$\mathrm{p}_{1}\left(\beta_{2}+2 \sqrt{\beta_{1} \beta_{3}}\right)^{-v_{1}}, \ldots, \mathrm{p}_{\mathrm{r}}\left(\beta_{2}+2 \sqrt{\beta_{1} \beta_{3}}\right)^{-v_{\mathrm{r}}}$
$\left[\left(\beta_{4}^{(1)}\right) ; \delta^{(1)}\right] ; \ldots ;\left[\left(\beta_{4}^{(\mathrm{r})}\right) ; \delta^{(\mathrm{r})}\right] ;$

\section{Proof}

In order to prove the given integral, we express the Fox's H-function and a general polynomials in the form of series and the H-function of several complex variables in terms of Mellin-Barnes contour integrals. Now interchanging the order of summations and integrations which is permissible under the stated conditions, we obtain 
$\sum_{\mathrm{G}=0}^{\infty} \sum_{\mathrm{g}=1}^{\mathrm{M}} \sum_{\mu_{1}=0}^{\left[\mathrm{N}_{1} / \mathrm{M}_{1}\right]} \ldots \sum_{\mu_{\mathrm{s}}=0}^{\left[\mathrm{N}_{\mathrm{s}} / \mathrm{M}_{\mathrm{s}}\right]} \frac{(-1)^{\mathrm{G}}\left(-\mathrm{N}_{1}\right)_{\mathrm{M}_{1} \mu_{1}}}{\mu_{1} !} \frac{\left(-\mathrm{N}_{\mathrm{s}}\right)_{\mathrm{M}_{\mathrm{s}} \mu_{\mathrm{s}}}}{\mu_{\mathrm{s}} !} \xi\left(\rho_{\mathrm{G}}\right)$

$\mathrm{A}\left[\mathrm{N}_{1} \mu_{1} ; \ldots ; \mathrm{N}_{\mathrm{s}} \mu_{\mathrm{s}}\right] \mathrm{v}_{1}^{\mu_{1}}, \ldots, \mathrm{v}_{\mathrm{s}}^{\mu_{\mathrm{s}}} \mathrm{A}\left(\mathrm{V} ; \mathrm{k}_{1}, \ldots, \mathrm{k}_{\ell}\right)$

$\frac{1}{(2 \pi \mathrm{i})^{\mu}} \int_{L_{1}} \ldots \int_{L_{r}} \psi\left(e_{1}, \ldots, e_{r}\right) \theta\left(e_{1}\right) \ldots \theta_{r}\left(r_{r}\right) p_{1}^{e_{1}} \ldots p_{r}^{e_{r}}$

$\left\{\int_{0}^{\infty} \mathrm{x}^{1-}\left(\mu-\mathrm{V} \rho_{\mathrm{n}}-\sum_{\mathrm{i}=1}^{\mathrm{s}} \mathrm{d}_{\mathrm{i}} \mu_{\mathrm{i}}-\sum_{\mathrm{i}=1}^{\ell} \mathrm{m}_{\mathrm{i}} \mathrm{k}_{\mathrm{i}}-v \mathrm{e}_{1} \ldots v_{\mathrm{r}} \mathrm{e}_{\mathrm{r}}\right)\right.$

$\left.\left(\beta_{1}+\beta_{2}+\beta_{3} x^{2}\right)^{\left.\mu-v \rho_{G}-\sum_{i=1}^{s} d_{i} \mu_{i}-\sum_{i=1}^{\ell} m_{i} h_{i}-v e_{1} \ldots v_{r} e_{r}\right)^{-3 / 2} d x d e_{1} \ldots d_{r}}\right\}$

Evaluating the above integral with the help of known theorem and reinterpreting the result in terms of $\mathrm{H}$ function of r-variables, we arrive at desired result.

\section{Numerical Results}

(a) Taking $\lambda=\mathrm{A}, \mathrm{u}^{(\mathrm{i})}=1, \mathrm{v}^{(\mathrm{i})}=\mathrm{B}^{(\mathrm{i})}$ and $\mathrm{D}^{(\mathrm{i})}=\mathrm{D}^{(\mathrm{i})}+1, \forall \mathrm{i} \in(1, \ldots, \mathrm{r})$, the result reduces to following integral transformation

$$
\begin{aligned}
& \int_{0}^{\infty} x^{1-\mu}\left(\beta_{1}+\beta_{2} x+\beta_{3} x^{2}\right)^{\mu-3 / 2} H_{P, Q}^{M, N}\left[\left(\frac{x}{\beta_{1}+\beta_{2} x+\beta_{3} x^{2}}\right)^{v} \mid \begin{array}{l}
\left(e_{P}, E_{P}\right) \\
\left(f_{Q}, F_{Q}\right)
\end{array}\right] \\
& . S_{N_{1}, \ldots, N_{s}}^{M_{1}, \ldots, M_{s}}\left[v_{1}\left(\frac{x}{\beta_{1}+\beta_{2} x+\beta_{3} x^{2}}\right)^{d_{1}}, \ldots, v_{s}\left(\frac{x}{\beta_{1}+\beta_{2} x+\beta_{3} x^{2}}\right)^{d_{s}}\right] \\
& \mathrm{S}_{\mathrm{v}}^{\mathrm{w}_{1}, \ldots, \mathrm{w}_{\ell}}\left[\mathrm{y}_{1}\left(\frac{\mathrm{x}}{\beta_{1}+\beta_{2}+\beta_{3} \mathrm{x}^{2}}\right)^{\mathrm{m}_{1}}, \ldots, \mathrm{y}_{\ell}\left(\frac{\mathrm{x}}{\beta_{1}+\beta_{2} \mathrm{x}+\beta_{3} \mathrm{x}^{2}}\right)^{\mathrm{m}_{\ell}}\right] \\
& \mathrm{F}_{\mathrm{C}: \mathrm{D}^{(1)} ; \ldots ; \mathrm{D}^{(\mathrm{r})}}^{\mathrm{A}: \mathrm{B}^{(1)}, \ldots \mathrm{B}^{(\mathrm{r})}}\left[-\mathrm{p}_{1}\left(\frac{\mathrm{x}}{\beta_{1}+\beta_{2} \mathrm{x}+\beta_{3} \mathrm{x}^{2}}\right)^{v_{1}}, \ldots,-\mathrm{p}_{\mathrm{r}}\left(\frac{\mathrm{x}}{\beta_{1}+\beta_{2} \mathrm{x}+\beta_{3} \mathrm{x}^{2}}\right)^{v_{\mathrm{r}}}\right] \\
& \cdot\left[\begin{array}{l}
{\left[1-\left(\beta_{1}\right): \theta^{(1)}, \ldots, \theta^{(\mathrm{r})}\right]:\left[1-\left(\beta_{2}^{(1)}\right): \gamma^{(1)}\right] ; \ldots ;\left[1-\left(\beta_{2}^{(\mathrm{r})}\right): \gamma^{(\mathrm{r})}\right]} \\
{\left[1-\left(\beta_{3}\right): \psi^{(1)} ; \ldots ; \psi^{(\mathrm{r})}\right]:\left[1-\left(\beta_{4}^{(1)}\right): \delta^{\prime}\right] ; \ldots ;\left[1-\left(\beta_{4}^{(\mathrm{r})}\right): \delta^{(\mathrm{r})}\right]}
\end{array}\right] \mathrm{dx} \\
& =\sqrt{\frac{\pi}{\beta_{3}}} \sum_{\mathrm{G}=0}^{\infty} \sum_{\mathrm{g}=1}^{\mathrm{m}} \sum_{\mu_{1}=0}^{\left[\mathrm{N}_{1} / \mathrm{M}_{1}\right]} \ldots \sum_{\mu_{\mathrm{s}}=0}^{\left[\mathrm{N}_{\mathrm{s}} / \mathrm{M}_{\mathrm{s}}\right]} \frac{(-1)^{\mathrm{G}}}{\mathrm{G} ! \mathrm{F}_{\mathrm{g}}} \frac{\left(-\mathrm{N}_{1}\right)_{\mathrm{M}_{1} \mu_{1}}}{\mu_{1} !} \ldots \frac{\left(-\mathrm{N}_{\mathrm{s}}\right)_{\mathrm{M}_{\mathrm{s}} \mu_{\mathrm{s}}}}{\mu_{\mathrm{s}} !} \xi\left(\rho_{\mathrm{G}}\right)
\end{aligned}
$$




$$
\begin{aligned}
& \mathrm{A}\left[\mathrm{N}_{1} \mu_{1} ; \ldots ; \mathrm{N}_{\mathrm{s}} \mu_{\mathrm{s}}\right] \mathrm{v}_{1}^{\mu_{1}}, \ldots, \mathrm{v}_{\mathrm{s}}^{\mu_{\mathrm{s}}} \mathrm{A}\left(\mathrm{V} ; \mathrm{k}_{1}, \ldots, \mathrm{k}_{\ell}\right) \\
& \left(\beta_{2}+\sqrt{\beta_{1} \beta_{3}}\right)^{\left(\mu-v \rho_{\mathrm{G}}-\sum_{\mathrm{i}=1}^{\delta} \mathrm{d}_{\mathrm{i}} \mu_{\mathrm{i}}-\sum_{\mathrm{i}=1}^{\ell} \mathrm{m}_{\mathrm{i}} \mathrm{k}_{\mathrm{i}}-1\right)}
\end{aligned}
$$

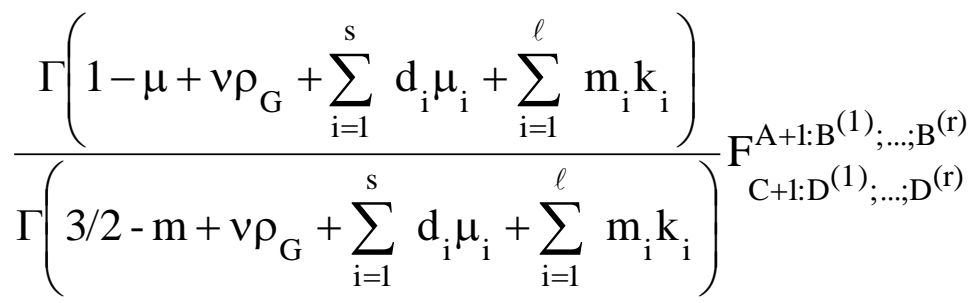

$$
\begin{aligned}
& {\left[-\mathrm{p}_{1}\left(\beta_{2}+2 \sqrt{\beta_{1} \beta_{3}}\right)^{-v_{1}}, \ldots,-\mathrm{p}_{\mathrm{r}}\left(\beta_{2}+2 \sqrt{\beta_{1} \beta_{3}}\right)^{-v_{\mathrm{r}}}\right]} \\
& {\left[1-\mu+\gamma \rho_{\mathrm{G}}+\sum_{\mathrm{i}=1}^{\mathrm{s}} \mathrm{d}_{\mathrm{i}} \mu_{\mathrm{i}}+\sum_{\mathrm{i}=1}^{\ell} \mathrm{m}_{\mathrm{i}} \mathrm{k}_{\mathrm{i}} ; v_{1} ; \ldots ; v_{\mathrm{r}}\right]\left[1-\left(\beta_{1}\right) ; \theta^{(1)} ; \ldots ; \theta^{(\mathrm{r})}\right]} \\
& {\left[1-\left(\beta_{3}\right): \psi^{(1)} ; \ldots ; \psi^{(\mathrm{r})}\right],\left[3 / 2-\mu+v \rho_{\mathrm{G}}+\sum_{\mathrm{i}=1}^{\mathrm{s}} \mathrm{d}_{\mathrm{i}} \mu_{\mathrm{i}}+\sum_{\mathrm{i}=1}^{\ell} \mathrm{m}_{\mathrm{i}} \mathrm{k}_{\mathrm{i}}: v_{1} ; \ldots ; v_{\mathrm{r}}\right]:} \\
& {\left[1-\left(\beta_{2}^{(1)}\right): \gamma^{(1)}\right] ; \ldots ;\left[1-\left(\beta_{2}^{(\mathrm{r})}\right): \gamma^{(\mathrm{r})}\right]} \\
& {\left[1-\left(\beta_{4}^{(1)}\right): \delta^{(1)}\right] ; \ldots ;\left[1-\left(\beta_{4}^{(\mathrm{r})}\right): \delta^{(\mathrm{r})}\right]}
\end{aligned}
$$

provided that $\operatorname{Re}\left(\beta_{1}\right)>0, \operatorname{Re}\left(\beta_{2}\right)>0, \beta_{3}>0$, the series on right side exist.

(b)Taking $\theta^{(1)}, \ldots, \theta^{(\mathrm{r})}=\gamma^{(1)}, \ldots, \gamma^{(\mathrm{r})}=\psi^{(1)}, \ldots, \psi^{(\mathrm{r})}=\delta^{(1)}, \ldots, \delta^{(\mathrm{r})}=v_{1}, \ldots, v_{\mathrm{r}}=\mu^{(1)}, \ldots, \mu^{(\mathrm{r})}$ in equation (2.1), we get the following integral transformation

$$
\begin{aligned}
& \int_{0}^{\infty} \mathrm{x}^{1-\mu}\left(\beta_{1}+\beta_{2} \mathrm{x}+\beta_{3} \mathrm{x}^{2}\right)^{\mu-3 / 2} \mathrm{H}_{\mathrm{P}, \mathrm{Q}}^{\mathrm{M}, \mathrm{N}}\left[\left(\frac{\mathrm{x}}{\beta_{1}+\beta_{2} \mathrm{x}+\beta_{3} \mathrm{x}^{2}}\right)^{v} \mid \begin{array}{c}
\left(\mathrm{e}_{\mathrm{P}}, \mathrm{E}_{\mathrm{P}}\right) \\
\left(\mathrm{f}_{\mathrm{Q}}, \mathrm{F}_{\mathrm{Q}}\right)
\end{array}\right] \\
& . \mathrm{S}_{\mathrm{N}_{1}, \ldots, \mathrm{N}_{\mathrm{s}}}^{\mathrm{M}_{1}, \ldots, \mathrm{M}_{\mathrm{s}}}\left[\mathrm{v}_{1}\left(\frac{\mathrm{x}}{\beta_{1}+\beta_{2} \mathrm{x}+\beta_{3} \mathrm{x}^{2}}\right)^{\mathrm{d}_{1}}, \ldots, \mathrm{v}_{\mathrm{s}}\left(\frac{\mathrm{x}}{\beta_{1}+\beta_{2} \mathrm{x}+\beta_{3} \mathrm{x}^{2}}\right)^{\mathrm{d}_{\mathrm{s}}}\right] \\
& \mathrm{S}_{\mathrm{V}}^{\mathrm{w}_{1}, \ldots, \mathrm{w}_{\ell}}\left[\mathrm{y}_{1}\left(\frac{\mathrm{x}}{\beta_{1}+\beta_{2}+\beta_{3} \mathrm{x}^{2}}\right)^{\mathrm{m}_{1}}, \ldots, \mathrm{y}_{\ell}\left(\frac{\mathrm{x}}{\beta_{1}+\beta_{2} \mathrm{x}+\beta_{3} \mathrm{x}^{2}}\right)^{\mathrm{m}_{\ell}}\right] \\
& \mathrm{G}^{0, \lambda:\left(\mathrm{u}^{(1)}, \mathrm{v}^{(1)}\right) ; \ldots ;\left(\mathrm{u}^{(\mathrm{r})}, \mathrm{v}^{(\mathrm{r})}\right)} \\
& \text { A:C: }\left[B^{(1)}, D^{(1)}\right] ; \ldots ;\left[B^{(r)}, D^{(r)}\right] \\
& {\left[\mathrm{p}_{1}^{\mu^{(1)}}\left(\frac{\mathrm{x}}{\beta_{1}+\beta_{2} \mathrm{x}+\beta_{3} \mathrm{x}^{2}}\right), \ldots, \mathrm{p}_{\mathrm{r}}^{\mu^{(\mathrm{r})}}\left(\frac{\mathrm{x}}{\beta_{1}+\beta_{2} \mathrm{x}+\beta_{3} \mathrm{x}^{2}}\right) \mid \begin{array}{l}
\left(\beta_{1}\right):\left(\beta_{2}^{(1)}\right) ; \ldots ;\left(\beta_{2}^{(\mathrm{r})}\right) \\
\left(\beta_{3}\right):\left(\beta_{4}^{(1)} ; \ldots ; \beta_{4}^{(\mathrm{r})}\right)
\end{array}\right] \mathrm{dx}}
\end{aligned}
$$




$$
\begin{aligned}
& =\sqrt{\frac{\pi}{\beta_{3}}} \sum_{\mathrm{G}=0}^{\infty} \sum_{\mathrm{g}=1}^{\mathrm{M}} \sum_{\mu_{1}=0}^{\left[\mathrm{N}_{1} / \mathrm{M}_{1}\right]} \ldots \sum_{\mu_{\mathrm{s}}=0}^{\left[\mathrm{N}_{\mathrm{s}} / \mathrm{M}_{\mathrm{s}}\right]} \frac{(-1)^{\mathrm{G}}}{\mathrm{G} ! \mathrm{F}_{\mathrm{g}}} \frac{\left(-\mathrm{N}_{1}\right)_{\mathrm{M}_{1} \mu_{1}}}{\mu_{1} !} \ldots \frac{\left(-\mathrm{N}_{\mathrm{s}}\right)_{\mathrm{M}_{\mathrm{s}} \mu_{\mathrm{s}}}}{\mu_{\mathrm{s}} !} \xi\left(\rho_{\mathrm{G}}\right) \\
& \mathrm{A}\left[\mathrm{N}_{1} \mu_{1} ; \ldots ; \mathrm{N}_{\mathrm{s}} \mu_{\mathrm{s}}\right] \mathrm{v}_{1}^{\mu_{1}}, \ldots, \mathrm{v}_{\mathrm{s}}^{\mu_{\mathrm{s}}} \mathrm{A}\left(\mathrm{V} ; \mathrm{k}_{1}, \ldots, \mathrm{k}_{\ell}\right) \\
& \left(\beta_{2}+\sqrt{\beta_{1} \beta_{3}}\right)^{\left.\mu-v \rho_{\mathrm{G}}-\sum_{\mathrm{i}=1}^{\mathrm{s}} \mathrm{d}_{\mathrm{i}} \mu_{\mathrm{i}}-\sum_{\mathrm{i}=1}^{\ell} \mathrm{m}_{\mathrm{i}} \mathrm{k}_{\mathrm{i}}-1\right)} \\
& \mathrm{G}^{0, \lambda:\left(\mathrm{u}^{(1)}, \mathrm{v}^{(1)}\right) \ldots, \ldots\left(\mathrm{u}^{(\mathrm{r})}, \mathrm{v}^{(\mathrm{r})}\right)} \\
& \text { A:C: }\left[B^{(1)}, D^{(1)}\right] ; \ldots ;\left[B^{(r)}, D^{(r)}\right] \\
& {\left[\mathrm{p}_{1}^{\frac{1}{\mu^{(1)}}}\left(\beta_{2}+2 \sqrt{\beta_{1} \beta_{3}}\right)^{-1}, \ldots, \mathrm{p}_{\mathrm{r}}^{\frac{1}{\mu^{(\mathrm{r})}}}\left(\beta_{2}+2 \sqrt{\beta_{1} \beta_{3}}\right)^{-1} \mid\right.} \\
& \left(\mu+v \rho_{\mathrm{G}}+\sum_{\mathrm{i}=1}^{\mathrm{s}} \mathrm{d}_{\mathrm{i}} \mu_{\mathrm{i}}+\sum_{\mathrm{i}=1}^{\ell} \mathrm{m}_{\mathrm{i}} \mathrm{k}_{\mathrm{i}}-1\right),\left(\beta_{1}\right):\left(\beta_{2}^{(1)}\right) ; \ldots ; \beta_{2}^{(\mathrm{r})} \\
& \left.\left(\beta_{3}\right),\left(\mu-v \rho_{\mathrm{G}}+\sum_{\mathrm{i}=1}^{\mathrm{s}} \mathrm{d}_{\mathrm{i}} \mu_{\mathrm{i}}+\sum_{\mathrm{i}=1}^{\ell} \mathrm{m}_{\mathrm{i}} \mathrm{k}_{\mathrm{i}}-1 / 2\right):\left(\beta_{4}^{(1)}\right) ; \ldots ;\left(\beta_{4}^{(\mathrm{r})}\right)\right]
\end{aligned}
$$

provided that $\operatorname{Re}\left(\beta_{1}\right)>0, \operatorname{Re}\left(\beta_{2}\right)>0, \beta_{3}>0 ; \mu^{(\mathrm{i})}>0(\mathrm{i}=1, \ldots, \mathrm{r}), 2\left(\mathrm{u}^{(\mathrm{i})}+\mathrm{v}^{(\mathrm{i})}\right)>$

$$
\left(\mathrm{A}+\mathrm{C}+\mathrm{B}^{(\mathrm{i})}+\mathrm{D}^{(\mathrm{i})}\right)
$$$$
\left|\arg \left(\mathrm{z}_{\mathrm{i}}\right)\right|<\left[\mathrm{u}^{(\mathrm{i})}+\mathrm{v}^{(\mathrm{i})}-\frac{\mathrm{A}}{2}-\frac{\mathrm{C}}{2}-\frac{\mathrm{B}^{(\mathrm{i})}}{2}-\frac{\mathrm{D}^{(\mathrm{i})}}{2}\right] \pi \text { and }
$$$$
v\left\{\min _{1 \leq \mathrm{j} \leq \mathrm{M}}\left[\operatorname{Re}\left(\mathrm{f}_{\mathrm{j}} / \mathrm{F}_{\mathrm{j}}\right)\right]\right\}+\sum_{\mathrm{i}=1}^{\mathrm{r}}\left\{\min _{1 \leq \mathrm{j} \leq \mathrm{u}^{(\mathrm{i})}}\left[\operatorname{Re}\left(\mathrm{d}_{\mathrm{j}}^{(\mathrm{i})}\right)\right]\right\}>\mu^{-2} \text {. }
$$

(c) When $\lambda=\mathrm{A}=\mathrm{C}=0$ in (2.1), we have the following transformation:

$$
\begin{aligned}
& \int_{0}^{\infty} \mathrm{x}^{1-\mu}\left(\beta_{1}+\beta_{2} \mathrm{x}+\beta_{3} \mathrm{x}^{2}\right)^{\mu-3 / 2} \mathrm{H}_{\mathrm{P}, \mathrm{Q}}^{\mathrm{M}, \mathrm{N}}\left[\left(\frac{\mathrm{x}}{\beta_{1}+\beta_{2} \mathrm{x}+\beta_{3} \mathrm{x}^{2}}\right)^{\mathrm{v}}\left(\begin{array}{c}
\left.\mathrm{e}_{\mathrm{P}}, \mathrm{E}_{\mathrm{P}}\right) \\
\left(\mathrm{f}_{\mathrm{Q}}, \mathrm{F}_{\mathrm{Q}}\right)
\end{array}\right]\right. \\
& . \mathrm{S}_{\mathrm{N}_{1}, \ldots, \mathrm{N}_{\mathrm{s}}}^{\mathrm{M}_{1}, \ldots, \mathrm{M}_{\mathrm{S}}}\left[\mathrm{v}_{1}\left(\frac{\mathrm{x}}{\beta_{1}+\beta_{2} \mathrm{x}+\beta_{3} \mathrm{x}^{2}}\right)^{\mathrm{d}_{1}}, \ldots, \mathrm{v}_{\mathrm{s}}\left(\frac{\mathrm{x}}{\beta_{1}+\beta_{2} \mathrm{x}+\beta_{3} \mathrm{x}^{2}}\right)^{\mathrm{d}_{\mathrm{s}}}\right] \\
& \mathrm{S}_{\mathrm{V}}^{\mathrm{W}_{1}, \ldots, \mathrm{w}_{\ell}}\left[\mathrm{y}_{1}\left(\frac{\mathrm{x}}{\beta_{1}+\beta_{2}+\beta_{3} \mathrm{x}^{2}}\right)^{\mathrm{m}_{1}}, \ldots, \mathrm{y}_{\ell}\left(\frac{\mathrm{x}}{\beta_{1}+\beta_{2} \mathrm{x}+\beta_{3} \mathrm{x}^{2}}\right)^{\mathrm{m}}\right]
\end{aligned}
$$




$$
\begin{aligned}
& \prod_{i=1}^{r} H_{B^{(i)}, D^{(i)}}^{(i)}\left[p_{i}^{(i)}\left(\frac{x}{\beta_{1}+\beta_{2} x+\beta_{3} x^{2}}\right)^{v_{i}} \mid \begin{array}{l}
\beta_{2}^{(i)}: \gamma^{(i)} \\
{\left[d^{(i)}: \delta^{(i)}\right.}
\end{array}\right] d x \\
& =\sqrt{\frac{\pi}{\beta_{3}}} \sum_{\mathrm{G}=0}^{\infty} \sum_{\mathrm{g}=1}^{\mathrm{M}} \sum_{\mu_{1}=0}^{\left[\mathrm{N}_{1} / \mathrm{M}_{1}\right]} \cdots \sum_{\mu_{\mathrm{s}}=0}^{\left[\mathrm{N}_{\mathrm{s}} / \mathrm{M}_{\mathrm{s}}\right]} \frac{(-1)^{\mathrm{G}}}{\mathrm{G} ! \mathrm{F}_{\mathrm{g}}} \frac{\left(-\mathrm{N}_{1}\right)_{\mathrm{M}_{1} \mu_{1}}}{\mu_{1} !} \ldots \frac{\left(-\mathrm{N}_{\mathrm{s}}\right)_{\mathrm{m}_{1} \alpha_{1}}}{\alpha_{1} !} \frac{\left(-\mathrm{n}_{\mathrm{s}}\right)_{\mathrm{M}_{\mathrm{s}} \mu_{\mathrm{s}}}}{\mu_{\mathrm{s}} !} \xi\left(\rho_{\mathrm{G}}\right) \\
& \mathrm{A}\left[\mathrm{N}_{1}, \mu_{1} ; \ldots ; \mathrm{N}_{\mathrm{s}}, \mu_{\mathrm{s}}\right] \mathrm{v}_{1}^{\mu_{1}} \ldots \mathrm{v}_{\mathrm{s}}^{\mu_{\mathrm{s}}} \mathrm{A}\left[\mathrm{V} ; \mathrm{k}_{1}, \ldots, \mathrm{k}_{\ell}\right] \\
& \left(\beta_{2}+2 \sqrt{\beta_{1} \beta_{3}}\right)\left(\mu-v \rho_{\mathrm{G}}-\sum_{\mathrm{i}=1}^{\mathrm{s}} \mathrm{d}_{\mathrm{i}} \mu_{\mathrm{i}}-\sum_{\mathrm{i}=1}^{\ell} \mathrm{m}_{\mathrm{i}} \mathrm{k}_{\mathrm{i}}-1\right) \\
& . \mathrm{H}_{1,1:\left[\mathrm{B}^{(1)}, \mathrm{D}^{(1)}\right] ; \ldots ;\left[\mathrm{B}^{(\mathrm{r})}, \mathrm{D}^{(\mathrm{r})}\right]}^{0,\left[\mathrm{u}^{(1)}{ }^{(1)}\right) \ldots ;(\mathrm{r})}\left[\begin{array}{c}
\mathrm{p}_{1}\left(\beta_{2}+2 \sqrt{\beta_{1} \beta_{3}}\right)^{-v_{1}} \\
\vdots \\
\mathrm{p}_{\mathrm{r}}\left(\beta_{2}+2 \sqrt{\beta_{1} \beta_{3}}\right)^{-v_{\mathrm{r}}}
\end{array} \mid\right. \\
& \left.\begin{array}{l}
\left.\left[\mu-v \rho_{\mathrm{G}}-\sum_{\mathrm{i}=1}^{\mathrm{s}} \mathrm{d}_{\mathrm{i}} \mu_{\mathrm{i}}-\sum_{\mathrm{i}=1}^{\ell} \mathrm{m}_{\mathrm{i}} \mathrm{k}_{\mathrm{i}}: v_{1} ; \ldots ; v_{\mathrm{r}}\right]:\left(\beta_{2}^{(1)}\right): \gamma^{(1)}\right] ; \ldots ;\left[\left(\beta_{2}^{(\mathrm{r})}\right): \gamma^{(\mathrm{r})}\right] \\
{\left[\mu-v \rho_{\mathrm{G}}-\sum_{\mathrm{i}=1}^{\mathrm{s}} \mathrm{d}_{\mathrm{i}} \mu_{\mathrm{i}}-\sum_{\mathrm{i}=1}^{\ell} \mathrm{m}_{\mathrm{i}} \mathrm{k}_{\mathrm{i}}-1 / 2: v_{1} ; \ldots ; v_{\mathrm{r}}\right]:\left[\left(\beta_{4}^{(1)}\right): \delta^{(1)}\right] ; \ldots ;\left[\left(\beta_{4}^{(\mathrm{r})}\right): \delta^{(\mathrm{r})}\right]}
\end{array}\right]
\end{aligned}
$$

valid under same conditions as obtained from (2.1).

(d) Replacing $\mathrm{N}_{1}, \ldots, \mathrm{N}_{\mathrm{s}}$ by $\mathrm{N}$ and $\mathrm{V} \rightarrow 0$, the result in (2.1) reduces to known result in [2] after little simplification.

(e) Taking $\mathrm{N}_{\mathrm{i}} \rightarrow 0(\mathrm{i}=1, \ldots, \mathrm{s}), \mathrm{V} \rightarrow 0, \beta_{\mathrm{i}}=0, \beta_{3}=1$, the result in (2.1) reduces to known result after little simplification obtained by Goyal and Mathur [4].

(f) If $\mathrm{r}=1$, and $\mathrm{M}_{\mathrm{i}}, \mathrm{N}_{\mathrm{i}} \rightarrow 0(\mathrm{i}=2, \ldots, \mathrm{s})$ and $\mathrm{V} \rightarrow 0$, the result in (2.1) reduces to known result with little modification recently derived by Gupta and Jain [5].

(g) Taking $\mathrm{V} \rightarrow 0$, the result in (2.1) reduces to known result after littler simplification obtained by Chaurasia and Shekhawat [2].

\section{Conclusion}

The integral thus obtained is believed to be one of the most general integral established so far. The findings of this paper are sufficiently general in nature and are capable of yielding numerous (known or new) results involving classical orthogonal polynomials hitherto scattered in the literature.

\section{References}

[1]. Braaksma, B.L.J., Asymptotic expansions and analytic continuations for a class of Barnes integrals, Compositio Math. 15 (1963), 239-341.

[2]. Chaurasia, V.B.L. and Shekhawat, Ashok Singh, An integral involving general polynomials and the H-function of several complex variables, Tamkang J. Math. 36(3) (2005), 255-260.

[3]. Chaurasia, V.B.L. and Godika, Anju, An integral involving certain product of special functions, Bull. Cal. Math. Soc. 91 (1999), 337-342.

[4]. Fox, C., The G and H-functions as symmetrical Fourier kernels, Trans. Amer. Math. Soc., 98, 395-429 (1961).

[5]. Goyal, S.P. and Mathur, S.L., On integrals involving H-function of two variables, Indian J. Pure \& Appl. Math. 7 (1976), 347-358.

[6]. Gupta, K.C. and Jain, R., An integral involving a general polynomial and product of Fox's H-function having general arguments, Ganita Sandesh, 3 (1989), 64-67.

[7]. Kilbas, A.A. and Ad Saigo, M., On the H-function, I. Appl. Math. Stoch. Anal., 12, 191-204(1999). 
[8]. Mathai, A.M. and Saxena, R.K., Theorems connecting L and $\mathrm{L}^{-1}$ and fractional integration operators, Proc. Nat. Acad. Sci., IndiaSect. A 45, 205-209 (1975)

[9]. Saxena, R.K., An integral involving G-function, Proc. Nat. Inst. Sci. India 26A (1960), 661-664.

[10]. Srivastava, H.M., A contour integral involving Fox's H-function, Indian J. Math. 14 (1972), 1-6.

[11]. Srivastava, H.M., A multilinear generating function for the Konhauser sets of bi-orthogonal polynomials suggested by the Laguerre polynomials, Pacific J. Math., 117 (1985), 183-191

[12]. Srivastava, H.M. and Panda, R., Some bilateral generating function for a class of generalized hypergeometric polynomials, J. Raine Angew. Math., 283/284 (1996), 265-274. 